\title{
A Retrospective Analysis of the Effects of Different Analgesics on the Pain and Functional Recovery of Patients with Traumatic Thoracolumbar Fractures in the Peri-Treatment Period
}

\section{Hao Yuan}

Fifth Affiliated Hospital of Zunyi Medical University

Quan-Yuan Chang

Southwest Medical University

Jie Chen

Southwest Medical University

\section{Ya-Ting Wang}

Southwest Medical University

\section{Zong-Jin Gan}

Southwest Medical University

\section{Song Wen}

Fifth Affiliated Hospital of Zunyi Medical University

\section{Ting-Ting Li}

Southwest Medical University

\section{Liu-Lin Xiong ( $0499465010 @ q q . c o m)$}

The Affiliated Hospital of Zunyi Medical University https://orcid.org/0000-0002-1623-5969

\section{Research article}

Keywords: traumatic thoracolumbar fracture, acetaminophen dihydrocodeine, celecoxib, etoricoxib, pain degree, network pharmacology

Posted Date: November 17th, 2020

DOl: https://doi.org/10.21203/rs.3.rs-103708/v1

License: (1) This work is licensed under a Creative Commons Attribution 4.0 International License. Read Full License 
Version of Record: A version of this preprint was published at Journal of Orthopaedic Surgery and Research on April 17th, 2021. See the published version at https://doi.org/10.1186/s13018-021-02401-w. 


\section{Abstract}

Background: There are few literatures analyze the effects of traumatic thoracolumbar vertebral fractures on prognosis pain and functional recovery from the perspective of analgesics used in the perioperative period, and lack of clinical guidance on the application of analgesics for its surgery.

Objective: To analyze the effects of peri-treatment analgesics on pain and recovery status of patients with thoracolumbar fractures combined with network pharmacology.

Methods: 719 patients with thoracolumar fractures were collected and divided into acetaminophen dihydrocodeine group ( $n=476)$, celecoxib group $(n=130)$ and etoricoxib group $(n=113)$ according to the use of analgesics. The visual analogue scale (VAS) was used to measure the degree of pain the number of days of the use of three analgesics, the postoperative Oswestry dysfunction index (ODI) scores and some Japanese orthopedics Association score (JOA) were recorded. The targets of three analgesics were obtained by Drugbank, and the target of acute pain caused by fracture is obtained by Genecards, then Venn diagram of the intersection genes was drawn through Venn. Protein interaction mapping (PPI) was obtained by using STRING database, the functional enrichment analysis of Gene Ontology (GO) and the enrichment analysis of Kyoto Encyclopedia of Genes and Genomics (KEGG) pathway were carried out based on Metascape database.

Results: The codeine group was higher than celecoxib and etoricoxib groups in the number of days of preoperative and postoperative analgesic use, VAS score difference on the preoperative and postoperative, and postoperative ODI index (all $\mathrm{P}<0.01$ ). There exist no statistically conspicuous differences among the three groups in basic characteristics, injury and intraoperative conditions, ODI score except ODI index, three JOA marks, and postoperative walking ability ( $P>0.05)$. Identify 3332 disease targets. PPI network analysis identified OPRM1, OPRK1, TRPV1, PTGS1 and PTGS2 as the core targets for acetaminophen dihydrocodeine in the treatment.

Conclusion: If multiple analgesic modes are adopted in the perioperative analgesic use, and the use of acetaminophen dihydrocodeine in combination with anti-inflammatory analgesics, the duration of analgesic use is expected to be shortened as far as possible to reduce the pain degree in postoperative recovery, providing a reference for the optimal use of anesthetics for clinical traumatic thoracolumbar fractures.

\section{Introduction}

Traumatic thoracolumbar fracture is a serious spinal injury that can involve the middle column, resulting in spinal instability, deformity, and nerve function injury. An epidemiological study showed that thoracolumbar fractures accounted for about $80 \%$ of spinal injuries $(56.09 \%$ of lumbar vertebrae, $23.77 \%$ of thoracic vertebrae), of which about $18 \%$ were burst fractures [1]. The main causes of injuries are highenergy injuries, such as traffic injuries, sports injuries, falling from high altitudes, etc. According to the survey, more than 160,000 people in North America are hospitalized for traumatic thoracolumbar 
fractures every year [2], with the rapid development of transportation and construction industry in China in recent years, the incidence of thoracolumbar fractures is increasing day by day. Every year, tens of thousands of people in China are deeply affected by thoracolumbar fractures, which is not conducive to social and economic development and people's happiness. Among them, the effect of pain treatment is a key factor that affects patients' functional recovery and a happy life. Therefore, the choice of analgesics during the peri-treatment period is particularly important.

In fracture surgery, acetaminophen dihydrocodeine celecoxib and etoricoxib are commonly used in clinical analgesics. Acetaminophen dihydrocodeine is a compound preparation composed mainly of acetaminophen and dihydrocodeine tartaric acid. Dihydrocodeine is a weak agonist for opioid receptors, similar to morphine and codeine in structure, but about 2 times stronger than codeine in analgesic effect, and is not easy to addiction, because it has the advantages of quick analgesic effect, good effect and so on, it is widely used for all kinds of pain, especially in the acute pain caused by fractures. But the disadvantage of its poor anti-inflammatory effect is also obvious $[3,4]$. Acetaminophen, which has analgesic and antipyretic effects, can selectively inhibit the biological production of prostaglandins in the central nervous system through the cyclooxygenase (COX) pathway. Meanwhile, its receptor selectivity for central COX-2 is 4.4 times that of COX-1. The results of Esmailian et al. illustrated that intravenous acetaminophen and morphine have the same therapeutic value in relieving fracture pain, and acetaminophen has a higher success rate of administration [5].

Celecoxib and etoricoxib, both non-steroidal anti-inflammatory drugs (NSAID), are selective cycox-2 inhibitors that reduce the synthesis of metabolites, including prostaglandin E2 (PGE2), prostaglandin I2 (PGI2), thromboxane A2 (TXA2), prostaglandin D2 (PGD2) and prostaglandin F2 (PGF2) [6], eventually inhibit these mediators and achieve anti-inflammatory analgesic, antipyretic function for traumatic fracture patients can reduce pain and inflammation for postoperative wound fast recovery have a positive impact, and the incidence of gastrointestinal adverse reactions caused by low [7].

From the effects of the above-mentioned analgesics, it can be understood that different types of analgesics have different analgesic effects on trauma and different effects on patients' recovery. Therefore, this article will focus on analyzing the effects of peri-treatment analgesics on the pain and recovery status of patients with thoracolumbar fractures after surgery, as well as analyzing their analgesic mechanisms through network pharmacology.

\section{Materials And Methods}

\subsection{Selection and grouping of patients}

A total of 719 patients with thoracolumbar fractures from November 2009 to January 2019 were collected from the Affiliated Hospital of Zunyi Medical University in Guizhou Province. All the patients were treated for thoracolumbar fractures by the same medical group under general anesthesia and underwent posterior unilateral laminectomy with small fenestration and intrathoracic bone grafting. The 
specific surgical methods include posterior pedicle screw distraction and reduction, unilateral spinal cord nerve decompression, and vertebral bone grafting and shaping. This study was approved by the Ethics Committee of the Affiliated Hospital of Zunyi Medical College (2015 Review No. (23))

Inclusion criteria: Patients with thoracolumbar fracture Tlics score $>4$ (Table 1) [8], under general anesthesia, the posterior unilateral lamina interlamellar space with small fenestration was performed for vertebroplasty in vivo. Exclusion criteria: absence of preoperative statistics from the injured segment.

According to the analgesic drugs used during the perioperative period, the patients were divided into three groups: the acetaminophen dihydrocodeine group $(n=476)$, the celecoxib group $(n=130)$, and the etoricoxib group $(n=113)$.

\subsection{Data Collection}

1. Basic information of the patient, including gender, age, height, weight, occupation and long-term residence;

2. Injury information, including the time of injury, main spinal segments of injury, preoperative Tlics score, preoperative Frankel grading and The visual analogue scale (VAS) score at rest;

3. Surgical information, including how long it took to start the operation after the injury (d), the date and year of the operation, the duration of the operation (h) and bleeding volume during operation.

4. The use of analgesics in the perioperative period;

5. 1 year follow-up after recovery, including postoperative VAS score when resting, how long can walk (d), each time walking distance $(\mathrm{m})$, Oswestry dysfunction index (ODI) score (the scale including pain intensity, self-reliance, extract, walking, sitting, standing, interfere with sleep, sex, social life and travel) of 10 aspects, such as ODI index and part of the Japanese orthopedics Association score (JOA) (lumbocrural pain, gait, and bladder function).

\subsection{Statistical methods}

The above data are classified into quantitative data and counting data according to data characteristics. Age, height, weight, BMI, injury after how long does it take to start the operation (d), preoperative Tlics score, operation time consuming $(\mathrm{h})$, intraoperative blood loss $(\mathrm{ml})$ and analgesic use preoperative postoperative VAS score, number of days and how long will it take to walk (d), each time walking distance $(\mathrm{m})$, ODI score, ODI index as the quantitative data of single factor analysis of variance, and use it in positive results for multiple comparison, using Bonferroni or Tamhane results to mean \pm standard deviation. Gender, age, body type, complications, occupation, altitude of long-term residence, cause of injury, number of injured segments, postoperative walking duration, walking distance and ODI index were grouped as counting data, and chi-square test was used to represent the results in the composition ratio of analgesics use. Rank sum test was used for Frankel classification, JOA lumbago, gait, and urine grade data. 
In addition, through [25] to search for acetaminophen dihydrocodeine tablets (paracetamol and Dihydrocodeine (PD)), celecoxib and etoricoxib's drug target. Through [26] to retrieve the target of acute pain caused by fracture, use [27] to draw the Venn diagram of the intersection gene. According to the intersection gene information, use the [28] database to obtain the Protein interaction mapping (PPI) of the intersection gene, and use the Metascape database to obtain the GO and KEGG of the target gene. Use Cytoscape to make a disease drug function interaction map. The final results are represented by Venn diagram, PPI, GO analysis, KEGG analysis, and disease drug function interaction diagram.

\section{Results}

\subsection{Basic characteristics of the patient}

There exist no statistical differences in gender, age, BIM, body type, complications, occupational and altitude sex, age, BIM, body type, complications, occupation and altitude among the three groups ( $p$ > 0.05, Fig. 1).

\subsection{Injury and condition assessment of the patient}

There exist no statistically conspicuous differences among the three groups in the cause of injury, number of injured segments, time from injury to operation, preoperative Tlics score, Frankel classification, operation time and intraoperative blood loss ( $P>0.05$, Fig. 2).

\subsection{The use time and pain assessment of analgesics in the peri-therapeutic period}

There were statistically significant differences in the number of days of use of analgesics and pain related indicators among the three groups. The number of days of use of analgesics before and after operation in the codeine group was significantly higher than that in the celecoxib and etoricoxib groups ( $8.8 \pm 0.98$ vs $5.05 \pm 0.37,8.8 \pm 0.98$ vs $5.60 \pm 0.54, \mathrm{P}<0.01$ ), and the celecoxib group was slightly lower than that in the etoricoxib group ( $5.05 \pm 0.37$ vs $5.60 \pm 0.54, P<0.01$, Fig. $2 \mathrm{~K})$.

VAS score of the codeine group was obviously higher than that of the other groups ( $8.10 \pm 0.33$ vs $6.99 \pm$ $0.09,8.10 \pm 0.33$ vs $7.12 \pm 0.13, P<0.01)$ one day before the operation, and there existed no statistical significance between the celecoxib and etoricoxib group $(P>0.05$, Fig. $2 L)$.

VAS scores of the codeine group at rest one month after surgery were higher than those of the celecoxib and etoricoxib group ( $1.64 \pm 0.77$ vs $1.12 \pm 0.75,1.64 \pm 0.77$ vs $1.15 \pm 0.72, P<0.01)$, but there remained no statistical significance between the celecoxib and etoricoxib group ( $P>0.05$, Fig. $2 \mathrm{M})$. 
The difference of VAS scores before and after operation in the codeine group was greater than that in the celecoxib and etoricoxib group. ( $6.45 \pm 0.87$ vs $5.98 \pm 0.75,6.45 \pm 0.87$ vs $5.87 \pm 0.73, P<0.01)$, there was no obvious difference between celecoxib and etoricoxib group $(P>0.05$, Fig. $2 N)$

\subsection{Recovery after 1 year of follow-up}

After the same surgery, the postoperative ODI score and JOA score, ODI personal life, extract, walk, sit, stand, sleep, sex, social life, the number of tourism, the ODI index section, ODI index grouping, JOA lumbocrural pain, gait, and postoperative bladder function and how long will it take to walk and postoperative walking distance there were no statistical significance $(P>0.05$, Fig. 3B-J, Fig. 3L, Fig. 3M, Fig. 4). In terms of ODI-1 pain score, the codeine group was significantly higher than the other two groups $(1.06 \pm 0.47$ vs $0.87 \pm 0.59,1.06 \pm 0.47$ vs $0.88 \pm 0.58, P<0.01)$, and the comparison between celecoxib and etoricoxib group was not significant $(P>0.05$, Fig. $3 A)$.

The ODI index of the codeine group was higher than that of the celecoxib group (14.44 \pm 4.35 vs $13.35 \pm$ $4.39, P<0.01)$, and there existed no obvious differences among the three groups $(P>0.05$, Fig. $3 K)$.

\subsection{Disease targets and drug targets and their Gene Ontology (GO) and Kyoto Encyclopedia of Genes and Genomics (KEGG) analyses}

Through network pharmacology, we will further analyze the targets of the above-mentioned drugs in the treatment of acute pain, and explain the characteristics of the above-mentioned analgesics from the analgesic mechanism. 3332 targets of acute pain caused by fracture were retrieved through Genecards, and 7 targets of acetaminophen and dihydrocodeine were obtained through Drugbank database, namely PTGES3, OPRD1, PTGS2, OPRK1, TRPV1, OPRM1, PTGS1; Celecoxib There are 5 targets, namely PDPK1, PTGS2, CDH11, CA2, CA3; 1 target of etoricoxib, namely PTGS2. According to the protein interaction map (PPI), OPRM1, OPRK1, TRPV1, PTGS1 and PTGS2 are directly related. Statistics show that acetaminophen and dihydrocodeine are mainly involved in neuropeptide binding, peroxidase activity, oxidoreductase activity, antioxidant activity, calcium channel activity, heme binding, calcium ion transmembrane transport protein activity, ion-gated channel activity and other biological processes. The main KEGG pathways are arachidonic acid metabolism, stimulation of interactions in nerve tissue, and serotonin-containing synapses. Celecoxib is mainly involved in biological processes such as carbon oxygen lyase activity, lyase activity, oxidoreductase activity, peroxidase activity, heme binding and tetrapyrrole binding. The main pathway of the drug KEGG is nitrogen metabolism. Etoricoxib is mainly involved in biological processes such as oxidoreductase activity, antioxidant activity, dioxygenase activity, heme binding and tetrapyrrole binding. The main pathways of KEGG related to the disease are VEGF, IL-17, NF-kappa B signaling pathway, tumor necrosis factor signaling pathway, and arachidonic acid metabolism. 


\section{Discussion}

It can be seen from the results that acetaminophen dihydrocodeine have been used for a longer duration of time within the peri-treatment analgesia of traumatic thoracolumbar fractures, the preoperative pain level is higher, and the pain improvement is relatively more during postoperative recovery, but the overall VAS score was still high after the operation, and the pain index was still high one year after the operation, and the functional recovery was poor. In addition, the timeliness of acetaminophen and dihydrocodeine is relatively poor, and an attempt is made to increase the use time of analgesics to greatly reduce pain. According to network pharmacology analysis, it is concluded that acetaminophen and dihydrocodeine mainly block central nerve pain by controlling calcium channels and so on. Celecoxib and etoricoxib play an analgesic effect mainly by controlling inflammation-related pathways and inhibiting the release of inflammatory factors. Therefore, it is believed that it may be the ability of celecoxib and etoricoxib to control inflammation, but not the ability of acetaminophen dihydrocodeine, that the clinical effect of postoperative pain and functional recovery in the acetaminophen dihydrocodeine group is worse than that in the other two groups.

\subsection{Analysis and discussion of case results}

This study found that acetaminophen dihydrocodeine may be used for a long time before surgery due to the lack of anti-inflammatory effects, which makes postoperative patients more painful. The pain caused by traumatic fracture is mainly caused by periosteum tear to the mechanical sensitive nerve fibers that supply the bone, bleeding at the broken end of the fracture, hyperemia and edema of local soft tissue to stimulate peripheral nerves and inflammatory pain, etc [9]. Acetaminophen dihydrocodeine is a compound preparation, acetaminophen has analgesic and antipyretic effects, but it is generally believed that it lacks anti-inflammatory effects [10]. Dihydrocodeine is metabolized into dihydromorphine, and the drug targets TRPV1, OPRM1H and OPRD1 mainly inhibit the release of neurotransmitters by controlling the voltage-gated calcium channel activity to reduce calcium ion current and increase potassium ion conductivity, thereby blocking the delivery of pain [11]. As an opioid alkaloid, acetaminophen dihydrocodeine, similar to morphine, mainly ACTS on the central nervous system. By binding to opioid receptors in the presynaptic membrane and postsynaptic membrane of pain-sensing nerve afferents, it inhibits the release of substance $P$ and thus blocks pain signals from being sent into the brain. The drug is effective in reducing the pain of nerve damage caused by fractures, but is less effective in treating inflammation caused by trauma. The main target of celecoxib, PTGS2, prevents the production of inflammatory prostaglandins by inhibiting the expression of COX-2 in the peripheral and central regions, inhibits pain hypersensitivity and achieves dual anti-inflammatory and analgesic effects. Peripheral tissue injury inhibits the production of inflammatory factors, reduces COX-2 mediated inflammatory response in the central center, increases the pain threshold, and inhibits the production of pain sensation $[12,13]$. Etoricoxib, also a COX-2 inhibitor drugs, has the same efficacy and safety as celecoxib, and the comparison of VAS scores of the two in the table showed no statistical difference. Therefore, acetaminophen double hydrogen codeine and celecoxib and relying on exam yesterday two non-steroidal anti-inflammatory drugs compared to its anti-inflammatory effect is poor, may result in traumatic 
thoracolumbar fractures especially after surgery incision healing process in the process of inflammation persists, so acetaminophen double hydrogen codeine in preoperative use of postoperative days were greater than the other two kinds of pain relievers. Our results suggest that clinical use of this drug should be cautious because its lack of anti-inflammatory effects can lead to persistent inflammation and increase the time of analgesic use. In clinical practice, it is not advisable to enhance the analgesic effect by increasing the duration of the usage of acetaminophen dihydrocodeine. Although it is not easy to become addicted to it, long-term use of analgesics may also cause patients to become addicted to it, and the drug has side effects on liver and kidney function.

\subsection{Network pharmacology analysis of analgesia mechanism}

In this paper, the network pharmacology analysis indicates that acetaminophen dihydrocodeine mainly blocks central nerve pain by controlling calcium channel and has no effect on fracture healing. Celecoxib and etoricoxib play an analgesic role by controlling inflammation-related pathways and inhibiting the release of inflammatory factors, and may have a positive effect on fracture healing.

In this study, all the three analgesics have PTGS2 common intersection targets with the acute pain caused by fracture, so PTGS2 is an important target for this acute pain. The PTGS2 protein, named peroxidase synthase 2 in prostaglandin, converts arachidonic acid into prostaglandin H2 (PGH2), which is an important step in the synthesis of prostaglandin. The double cyclooxygenase and peroxidase in the prostaglandins biosynthesis pathway are a kind of C20 lipoproteins mainly derived from arachidonic acid, which have a special role in inflammatory response [14-16]. In addition, in the synthesis process, 2arachidonic acid glyceride is metabolized to produce $\mathrm{PGH} 2$ glyceride, which can lead to pain response [17]. Cyclooxygenase activity oxidized arachidonic acid to intracorporate prostagtin G2 (PGG2), and peroxidase activity reduced PGG2 to intracorporate peroxidase PGH2 $[18,19]$. The binding site of NonSteroidal Antiinflammatory Drugs (NSAIDS) corresponds to the active site of cyclooxygenase.

GO enrichment analysis confirmed that acetaminophen dihydrocodine mainly regulates neuropeptide binding, peroxidase activity, redox enzyme activity, calcium channel activity, heme binding, calcium ion transmemmeteral transporter activity and other biological processes to block the transmission of pain signals in the central nervous system.

CA2, which is essential for bone resorption and osteoclast differentiation at the celecoxib intersection target, is analgesic, anti-inflammatory and beneficial to fracture healing. Celecoxib and etoricoxib are mainly involved in biological processes such as redox activity, antioxidant activity, dioxidase activity, heme binding and tetrapyrrole binding. The main pathways of KEGG associated with the disease were VEGF, IL-17, NF-Kappa B, TNF signaling pathway, and arachidonic acid metabolism. VEGF signaling pathway is the upstream pathway of the MAPK signaling pathway, which controls its activation or not. MAPK signaling pathway plays a vital role in growth reproduction, cell differentiation, cell movement and other cell activities, and is one of the important pathways in the dynamic balance of bone development and bone metabolism, and is involved in the regulation of osteoblasts' metabolism and inflammatory 
response [20]. The activation of MAPK signaling pathway can accelerate the maturation cycle of osteoblasts, stimulate the release of alkaline phosphatase, and accelerate the deposition of calcium, which is conducive to fracture healing [21]. Moreover, the activation of VEGF signaling pathway is also conducive to the regeneration of blood vessels and the reconstruction of blood circulation in the fractured part [22]. IL-17 signaling pathway is a pro-inflammatory pathway, which is related to various biological activities such as inflammatory development and immune response, and can induce the differentiation and maturation of pre-osteoblastic cells independent of GSK-3 cell/catenin activity [23]. The NF-Kappa B signaling pathway regulates genes involved in immunity, inflammation, and cell survival [24]. Therefore, it can be known that celecoxib and etoricoxib are mainly involved in cellular immune response, inflammatory response and bone cell metabolism to relieve the pain of traumatic fracture and achieve good postoperative fracture recovery with low pain degree. Hence, the results of network pharmacology analysis can explain the phenomenon that the therapeutic effect of acetaminophen dihydrocodeine in clinical retrospective analysis is better than celecoxib and etoricoxib.

This study retrospectively analyzed the effect of analgesics on postoperative recovery of 719 thoracolumbar fracture patients in the Affiliated Hospital of Zunyi Medical University in Guizhou Province from November 2009 to January 2019, and the analgesic mechanism was analyzed by combining network pharmacology. The results manifested that if the multi-unit analgesic mode is used during the use of analgesics in the peri-treatment period, and the use of acetaminophen dihydrocodeine in combination with anti-inflammatory analgesics, it is expected to shorten the use time of analgesics as much as possible to reduce postoperative. The degree of pain during recovery provides a reference for the high-quality use of anesthetics for clinical traumatic thoracolumbar fractures.

\section{Abbreviations}

\section{VAS}

The visual analogue scale

ODI

Oswestry dysfunction index

JOA

Japanese orthopedics Association score

PPI

Protein interaction mapping

GO

Gene ontology

KEGG

Kyoto Encyclopedia of Genes and Genomics

coX

Cyclooxygenase

NSAID

Non-steroidal anti-inflammatory drugs 
PG

prostaglandin

TXA2

thromboxane A2

PD

Paracetamol and dihydrocodeine

VEGF

vascular endothelial growth factor

CA2

Carbonic anhydrase 2

IL-17

Interleukin-17

TNF

Tumor necrosis factor

NF-Kappa B

Nuclear factor NF-kappa-B

MAPK

Mitogen-activated protein kinase

NSAIDS

Non-Steroidal Antiinflammatory Drugs

\section{Declarations}

\section{Ethics approval and consent to participate}

This study was approved by the Ethics Committee of the Affiliated Hospital of Zunyi Medical College (2015 Review No. (23))

\section{Consent for publication}

Consent for publication was obtained from all participants.

\section{Availability of data and materials}

The data are available from the corresponding author upon reasonable request.

\section{Competing interests}

The authors declare that they have no conflict of interests.

\section{Funding}

This study was supported by National Natural Science Foundation of China (No. 82060243). 


\section{Authors' contributions}

Y.H. developed the idea for the study, Y.H. and L.T.T. did the analyses, C.Q.Y. and L.T.T. wrote the paper.all authors contributed to the writing and revisions.

\section{Acknowledgements}

Not applicable.

\section{Authors' information (optional)}

\section{Affiliations}

Department of Anesthesiology, The Affiliated Hospital of Zunyi Medical University, Zunyi 563000, Guizhou, China.

Yuan Hao \& Xiong Liulin

National Traditional Chinese Medicine Clinical Research Base and Western Medicine Translational Medicine Research Center, South West Medical University, Luzhou 646000, China

Chang Quanyuan, Li Tingting, Chen Jie \& Wang Yating

\section{References}

1. Liu P, Yao Y, Liu MY, Fan WL, Chao R, Wang ZG, Liu YC, Zhou JH, Zhao JH. Spinal trauma in mainland China from 2001 to 2007: an epidemiological study based on a nationwide database. Spine. 2012;37(15):1310-5.

2. Wood KB, Li W, Lebl DR, Ploumis A. Management of thoracolumbar spine fractures. The spine journal: official journal of the North American Spine Society. 2014;14(1):145-64.

3. Edwards JE, McQuay HJ, Moore RA. Single dose dihydrocodeine for acute postoperative pain. Cochrane Database Syst Rev. 2000;2000(4):Cd002760.

4. Graham GG, Scott KF. Mechanism of action of paracetamol. Am J Ther. 2005;12(1):46-55.

5. Esmailian M, Moshiri R, Zamani M. Comparison of the Analgesic Effect of Intravenous Acetaminophen and Morphine Sulfate in Rib Fracture; a Randomized Double-Blind Clinical Trial. Emergency (Tehran Iran). 2015;3(3):99-102.

6. Li W, Zhang Y, Liu Y, Yue F, Lu Y, Qiu H, Gao D, Gao Y, Wu Y, Wang Z, et al. In vitro kinetic evaluation of the free radical scavenging ability of propofol. Anesthesiology. 2012;116(6):1258-66.

7. Krasselt M, Baerwald C. Celecoxib for the treatment of musculoskeletal arthritis. Expert opinion on pharmacotherapy. 2019;20(14):1689-702.

8. Pneumaticos SG, Karampinas PK, Triantafilopoulos G, Koufos S, Polyzois V, Vlamis J. Evaluation of TLICS for thoracolumbar fractures. European spine journal: official publication of the European 
Spine Society the European Spinal Deformity Society the European Section of the Cervical Spine Research Society. 2016;25(4):1123-7.

9. Mitchell SAT, Majuta LA, Mantyh PW. New Insights in Understanding and Treating Bone Fracture Pain. Curr Osteoporos Rep. 2018;16(4):325-32.

10. Meredith TJ, Goulding R. Paracetamol. Postgraduate medical journal. 1980;56(657):459-73.

11. Wu J, Yan N, Yan Z. Structure-Function Relationship of the Voltage-Gated Calcium Channel Ca(v)1.1 Complex. Adv Exp Med Biol. 2017;981:23-39.

12. Kondreddy VK, Kamatham AN. Celecoxib, a COX-2 inhibitor, synergistically potentiates the antiinflammatory activity of docosahexaenoic acid in macrophage cell line. Immunopharmacol Immunotoxicol. 2016;38(2):153-61.

13. Puljak L, Marin A, Vrdoljak D, Markotic F, Utrobicic A, Tugwell P. Celecoxib for osteoarthritis. Cochrane Database Syst Rev. 2017;5(5):Cd009865.

14. Barnett J, Chow J, Ives D, Chiou M, Mackenzie R, Osen E, Nguyen B, Tsing S, Bach C, Freire J, et al: Purification, characterization and selective inhibition of human prostaglandin $\mathrm{G} / \mathrm{H}$ synthase 1 and 2 expressed in the baculovirus system. Biochimica et biophysica acta 1994, 1209(1):130-139.

15. Lucido MJ, Orlando BJ, Vecchio AJ, Malkowski MG. Crystal Structure of Aspirin-Acetylated Human Cyclooxygenase-2: Insight into the Formation of Products with Reversed Stereochemistry. Biochemistry. 2016;55(8):1226-38.

16. Landino LM, Crews BC, Gierse JK, Hauser SD, Marnett LJ. Mutational analysis of the role of the distal histidine and glutamine residues of prostaglandin-endoperoxide synthase-2 in peroxidase catalysis, hydroperoxide reduction, and cyclooxygenase activation. J Biol Chem. 1997;272(34):21565-74.

17. Musee J, Marnett LJ. Prostaglandin H synthase-2-catalyzed oxygenation of 2-arachidonoylglycerol is more sensitive to peroxide tone than oxygenation of arachidonic acid. J Biol Chem. 2012;287(44):37383-94.

18. Kulmacz RJ, Wang LH. Comparison of hydroperoxide initiator requirements for the cyclooxygenase activities of prostaglandin H synthase-1 and - 2. J Biol Chem. 1995;270(41):24019-23.

19. Kim SF, Huri DA, Snyder SH. Inducible nitric oxide synthase binds, S-nitrosylates, and activates cyclooxygenase-2. Science. 2005;310(5756):1966-70.

20. Sun Y, Liu WZ, Liu T, Feng X, Yang N, Zhou HF. Signaling pathway of MAPK/ERK in cell proliferation, differentiation, migration, senescence and apoptosis. J Recept Signal Transduct Res. 2015;35(6):600-4.

21. Majidinia M, Sadeghpour A, Yousefi B. The roles of signaling pathways in bone repair and regeneration. Journal of cellular physiology. 2018;233(4):2937-48.

22. Hamilton JL, Nagao M, Levine BR, Chen D, Olsen BR, Im HJ. Targeting VEGF and Its Receptors for the Treatment of Osteoarthritis and Associated Pain. Journal of bone mineral research: the official journal of the American Society for Bone Mineral Research. 2016;31(5):911-24. 
23. Wang Y, Kim J, Chan A, Whyne C, Nam D. A two phase regulation of bone regeneration: IL-17F mediates osteoblastogenesis via C/EBP- $\beta$ in vitro. Bone. 2018;116:47-57.

24. Lawrence T. The nuclear factor NF-kappaB pathway in inflammation. Cold Spring Harb Perspect Biol. 2009;1(6):a001651.

25. Drugbank. https://www.drugbank.ca/drugs/. Accessed 20 August 2020.

26. Genecards. https://www.genecards.org. Accessed 20 August 2020.

27. Venn. http://bioinformatics.psb.ugent.be/webtools/Venn/. Accessed 21 August 2020.

28. STRING database. https://string-db.org/cgi/input.pl. Accessed 21 August 2020.

\section{Tables}

Table 1 Tlics standard scale

\begin{tabular}{|c|c|c|}
\hline Type & & Point \\
\hline \multirow[t]{4}{*}{ Injury morphology } & Compression & 1 \\
\hline & Burst & 2 \\
\hline & Translation/rotation & 3 \\
\hline & Distraction & 4 \\
\hline \multirow{3}{*}{$\begin{array}{l}\text { Posterior } \\
\text { ligamentous }\end{array}$} & Intact & 0 \\
\hline & Suspeted/indtermninate & 2 \\
\hline & Injured & 3 \\
\hline \multirow[t]{5}{*}{ Neurologic status } & Intact & 0 \\
\hline & Nerve root & 2 \\
\hline & Cord, conus medullaris, complete & 2 \\
\hline & Cord, conus medullaris, incomplete & 3 \\
\hline & Cauda equine & 3 \\
\hline \multirow{3}{*}{$\begin{array}{l}\text { Treatment options } \\
\text { (total score) }\end{array}$} & Nonpenative treatment & $\leq 3$ \\
\hline & Nonoperative or surgery & 4 \\
\hline & Surgial intervention & $\geq 5$ \\
\hline
\end{tabular}

\section{Figures}



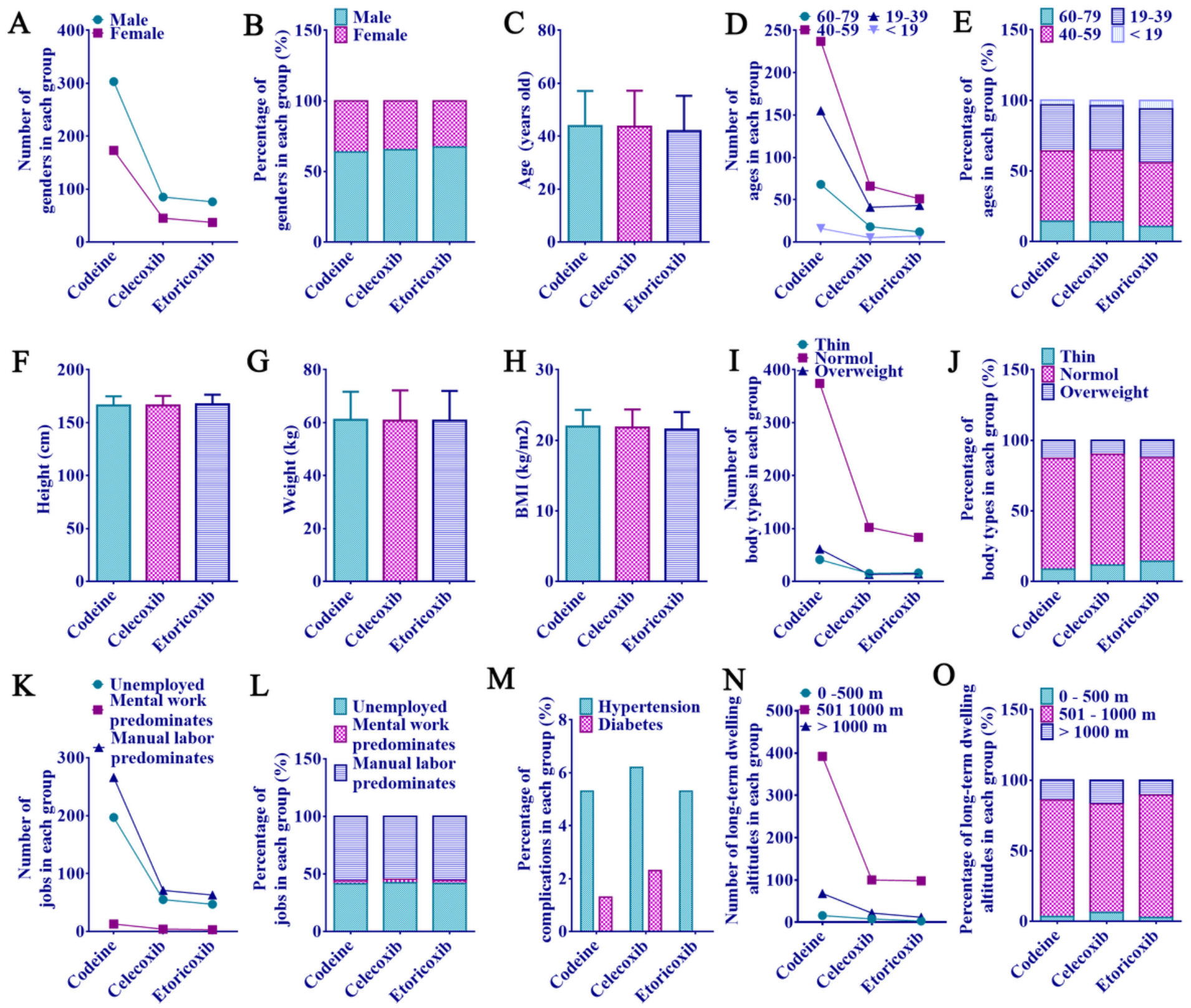

Figure 1

Basic features of the patient A. Number of different genders; B. Percentage by sex; C. Age value; D. Number of age groups; E. Percentage of different age groups; F. Height value; G. Body weight; $\mathrm{H}$. BMI value; I. Number of different body types; J. Percentage of different body types; K. Number of different occupations; L. Percentage of different occupations; M. Percentage of different comorbidities; N. Number of groups of different altitudes of long-term residence; $\mathrm{O}$. Percentage of groups with different altitudes of long-term residence 

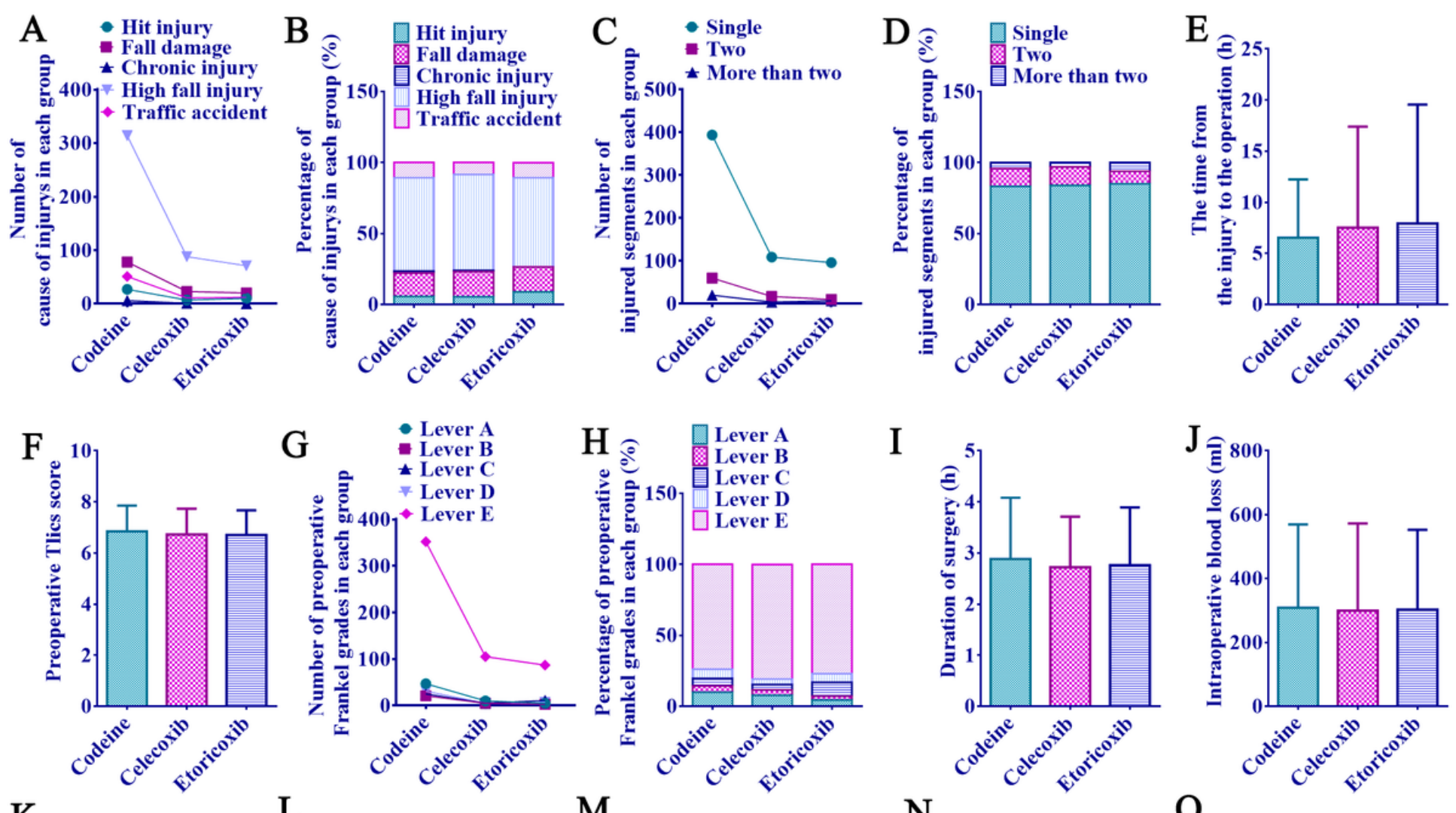

I
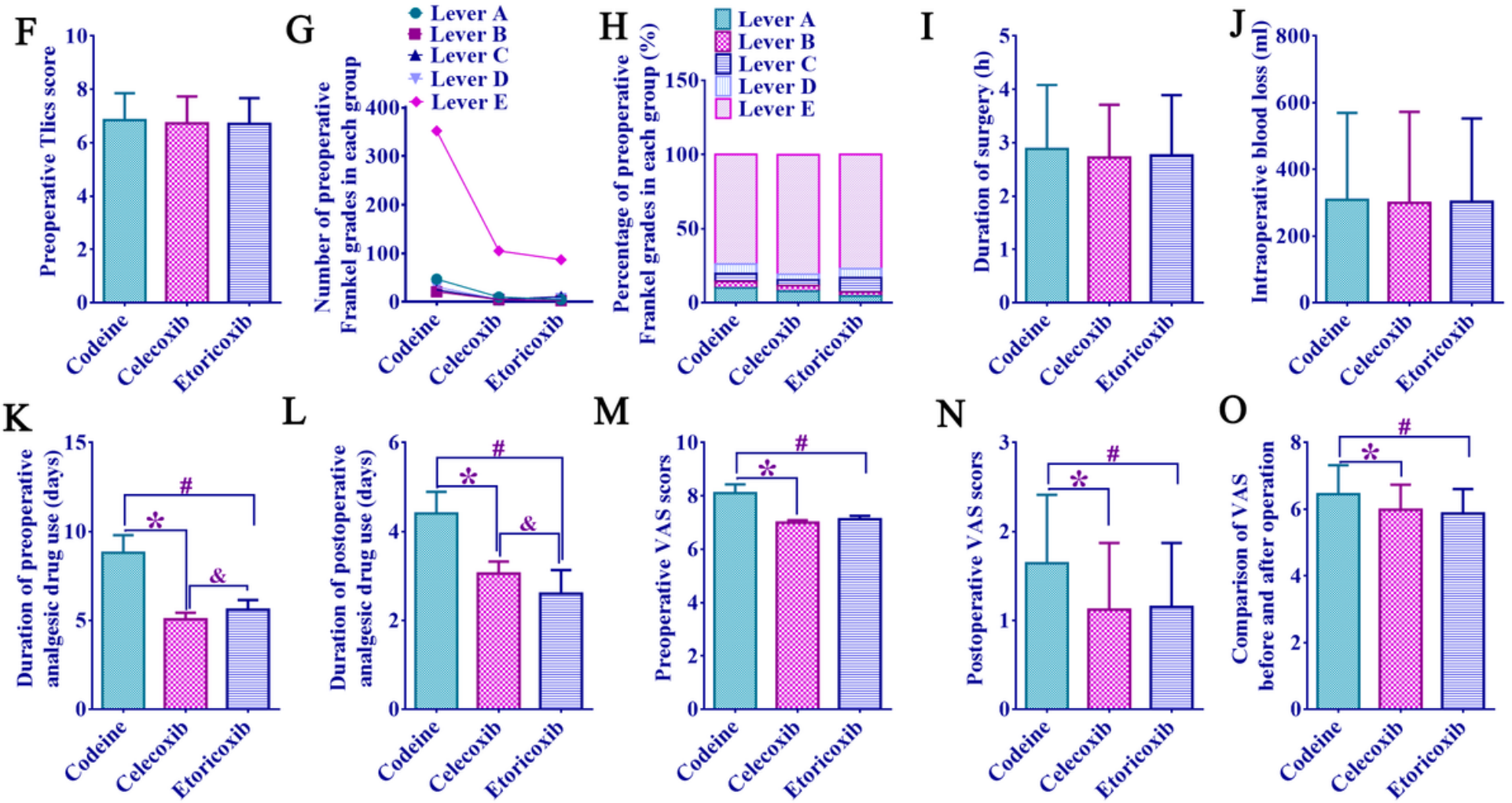

M

$\mathrm{N}$
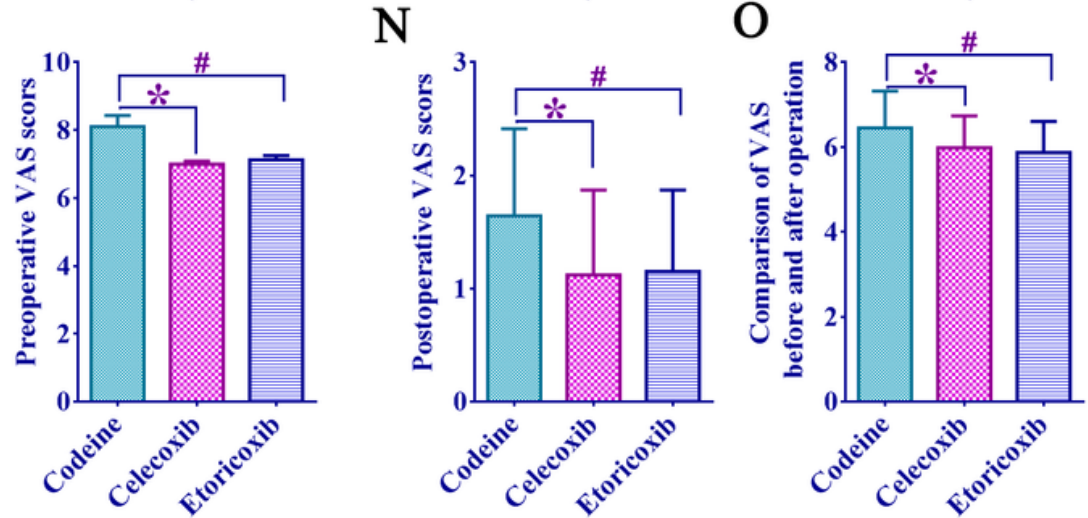

Figure 2

Assessment of the patient's injury and condition, duration of perioperative analgesics and pain score A. The number of different injury causes; $B$. Percentage of different causes of injury; $C$. The number of different injured segments; D. Percentage of different injured segments; $E$. Time from injury to operation (days); F. Preoperative Tlics score; G. The number of different Frankel grades before surgery; $\mathrm{H}$.

Preoperative percentages of different Frankel grades; I. Operation time (hours); J. Intraoperative blood loss (ml); K. Time of preoperative analgesic drug use (days); L. Use time of postoperative analgesics (days); M. VAS during braking one day before surgery; N. VAS rest assessment one month after surgery; 0. Comparison of VAS differences before and after operation *Vs Codeine group, significant difference, \#vs Celecoxib group, significant difference, $\mathrm{P}<0.01$ 
A
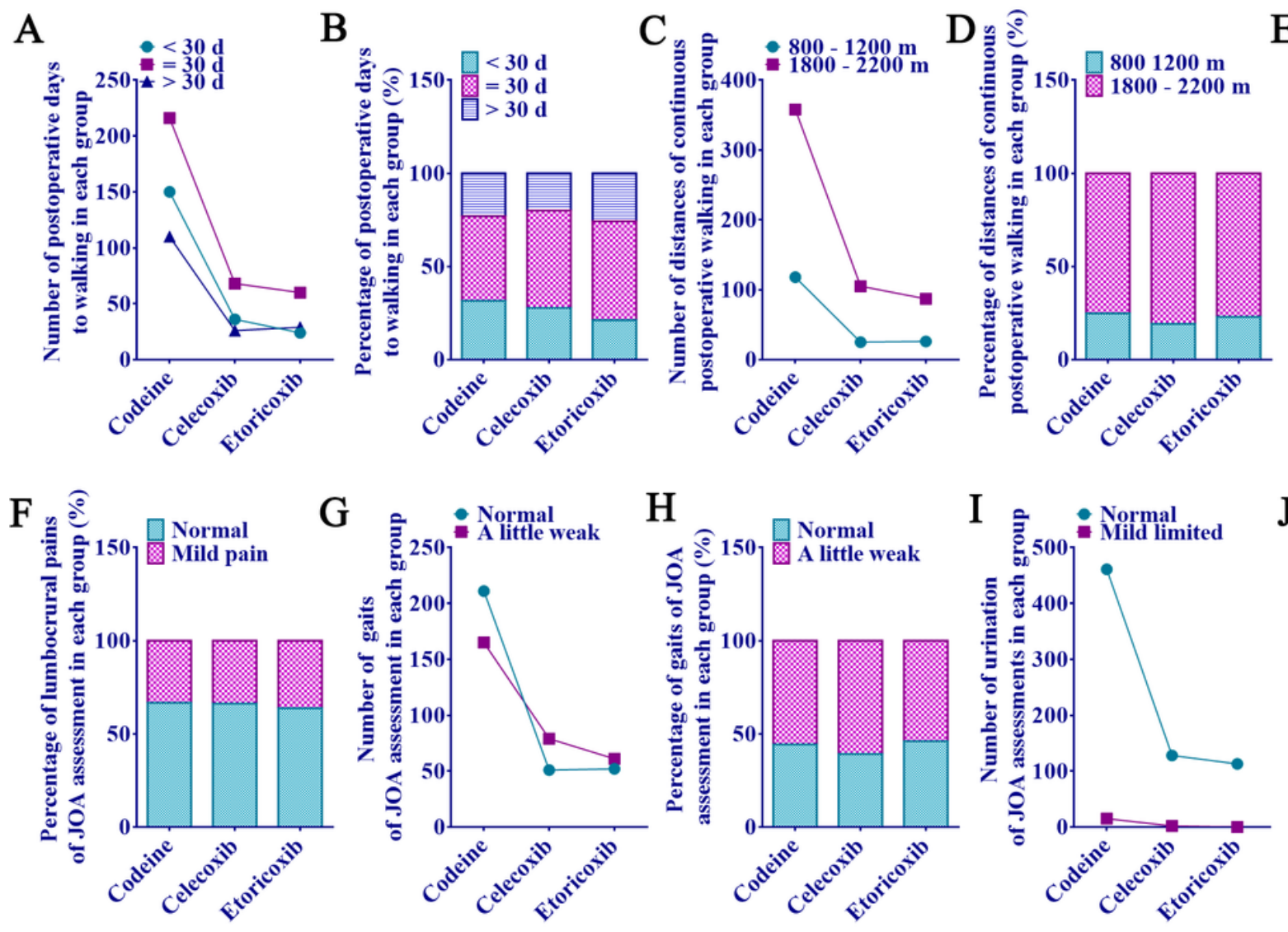

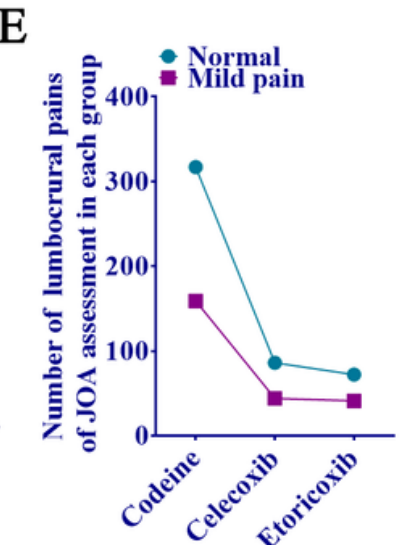

\section{Figure 3}

Postoperative ODI score A. ODI-1 (pain assessment); B. ODI-2 (personal life); C. ODI-3 (weight lifting); D. ODI-4 (walking); E. ODI-5 (seated); F. ODI-6 (walking); G. ODI-7 (Sleep); H. ODI-8 (Sex life); I. ODI-9 (Social life); J. ODI-10 (Travel); K. ODI index number; L. The number of segments of different ODI indexes; M. Percentage of different ODI index segments. * There were significant differences between the codeine group and the celecoxib group, and there were significant differences between the codeine group and the etoricoxib group, $\mathrm{P}<0.01$ 

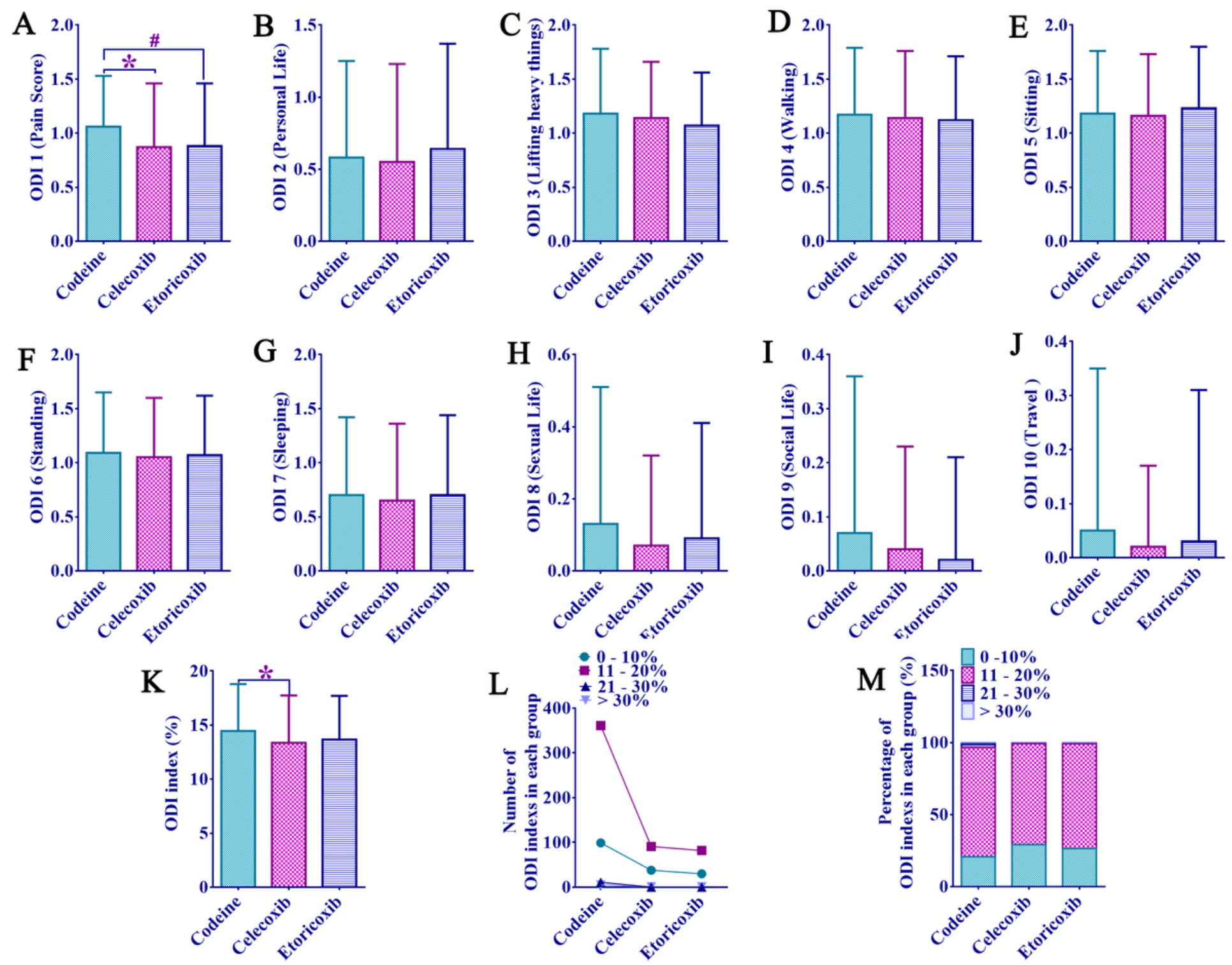

Figure 4

Postoperative walking condition and JOA score A. The number of different days from postoperative to walking; B. Percentage of different days from postoperative to walking; $C$. The number of different distances walked after surgery; $D$. The percentage of different distances walked after surgery; $E$. The number of different states of waist and leg pain; F. JOA percentage of different states of lumbago and leg pain; G. JOA the number of different states of gait; $\mathrm{H}$. JOA the percentage of different states of gait; I. The amount of JOA urine in different states; J. JOA the percentage of different states of urination 


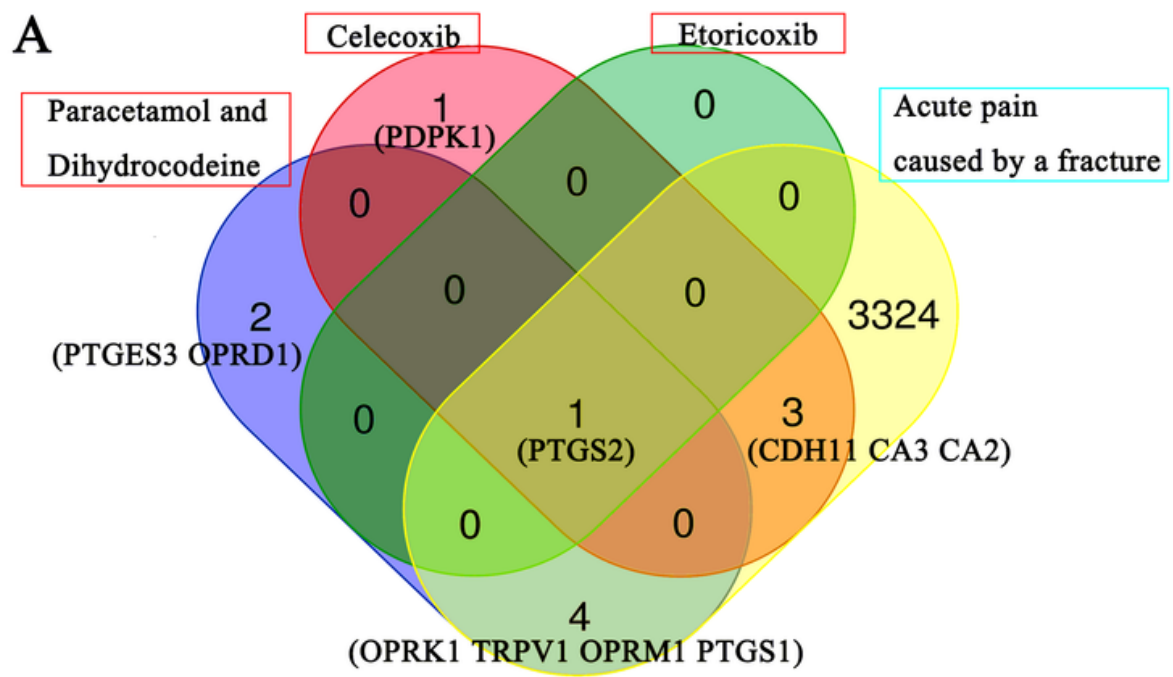

B

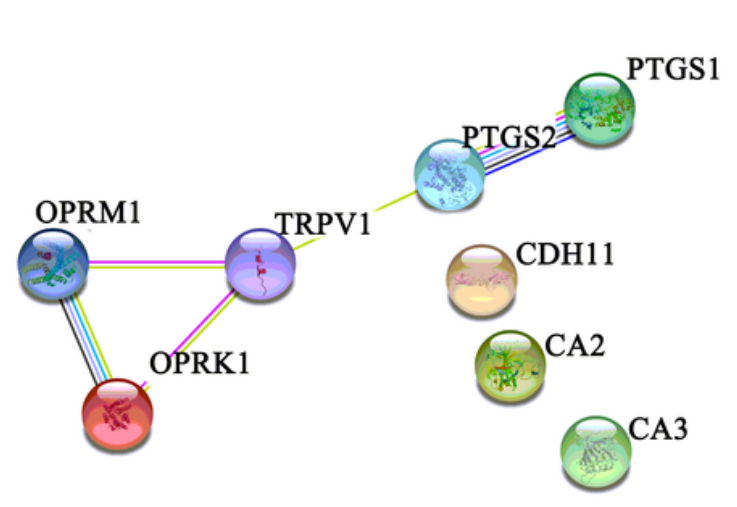

Figure 5

Network pharmacology analysis of related analgesics A. Venn diagram; B. PPI
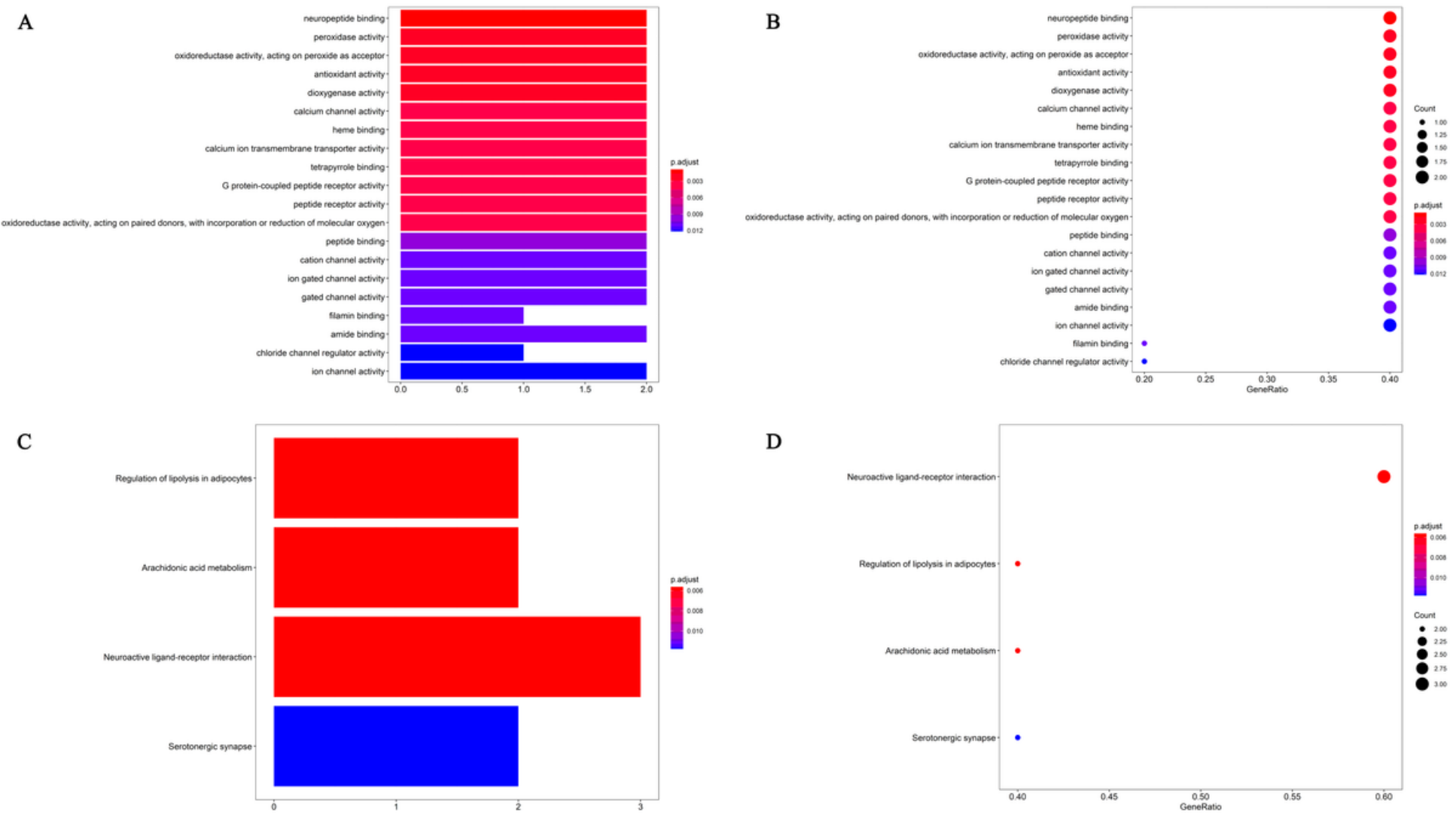

Figure 6

GO and KEGG enrichment analysis of targets at the intersection of aminophenol dihydrocodeine and disease A. GO enrichment analysis histogram; B. Go enrichment analysis bubble diagram; C. KEGG enrichment analysis bar chart; D. KEGG enrichment analysis bubble diagram 
A

C
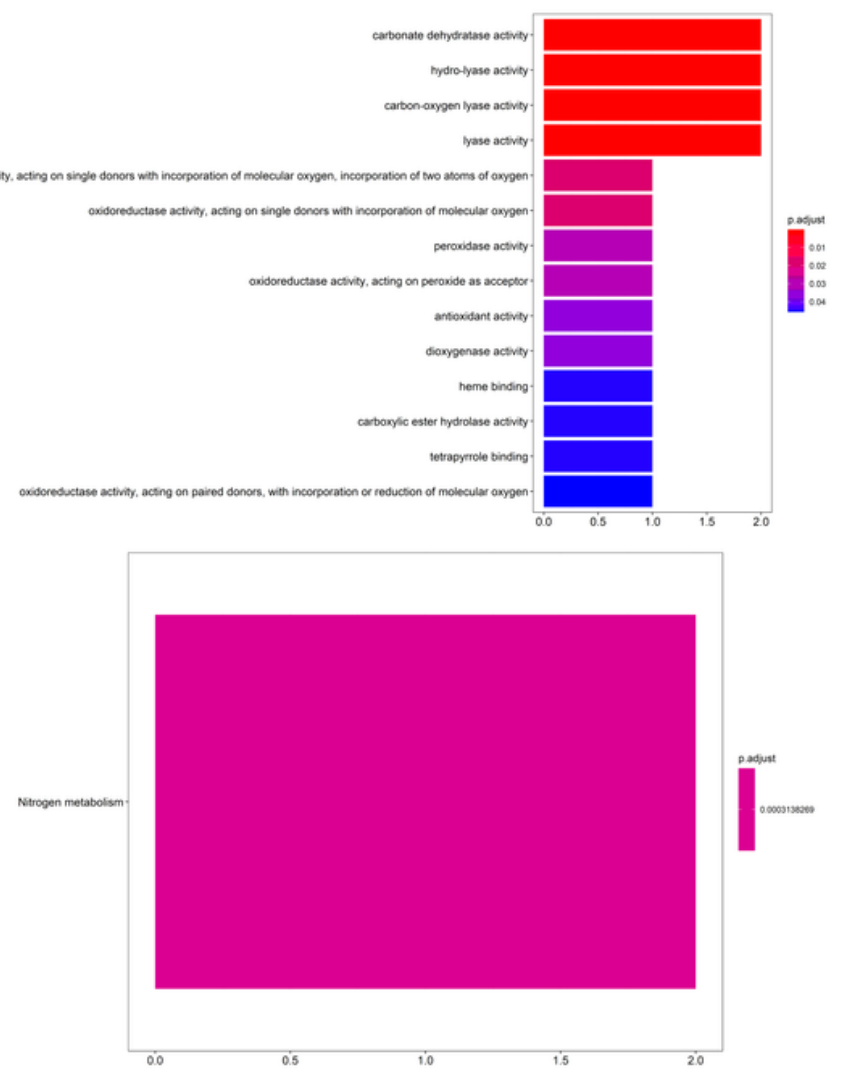

B

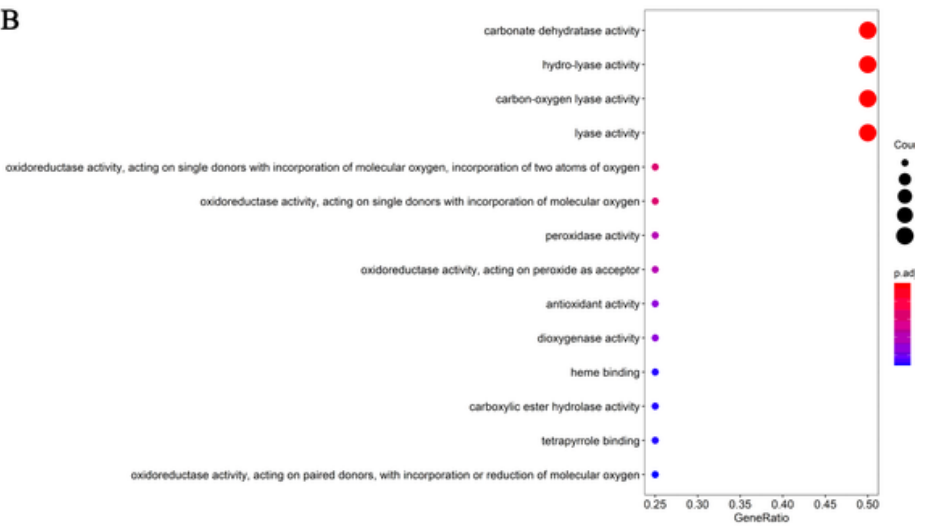

D

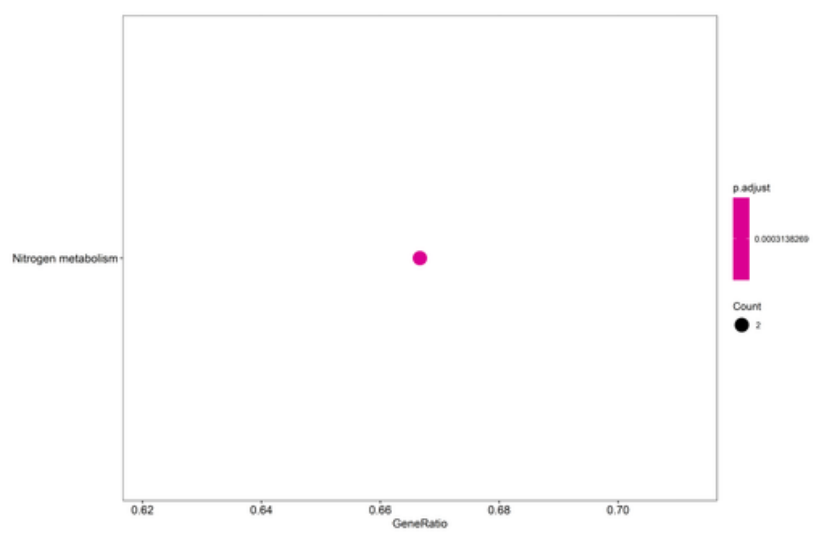

Figure 7

GO and KEGG enrichment analysis of targets at the intersection of celecoxib and A. GO enrichment analysis histogram; B. Go enrichment analysis bubble diagram; C. KEGG enrichment analysis bar chart; D. KEGG enrichment analysis bubble diagram 
A

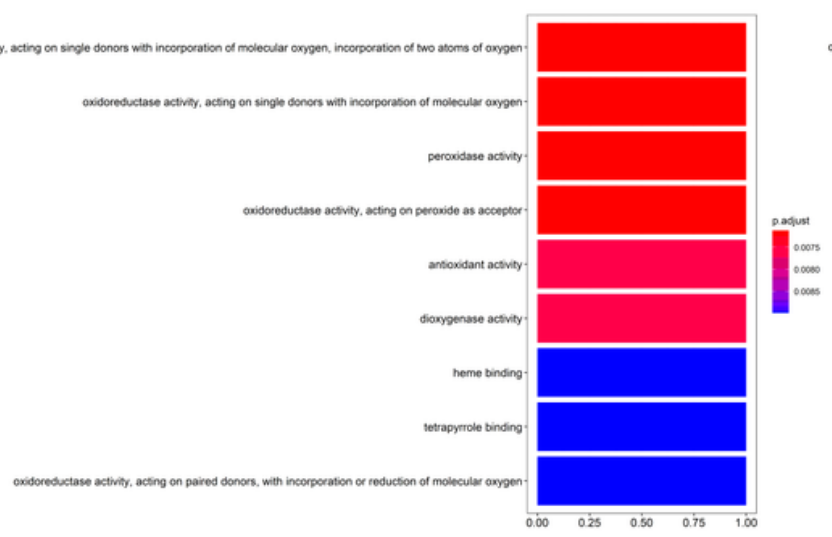

B

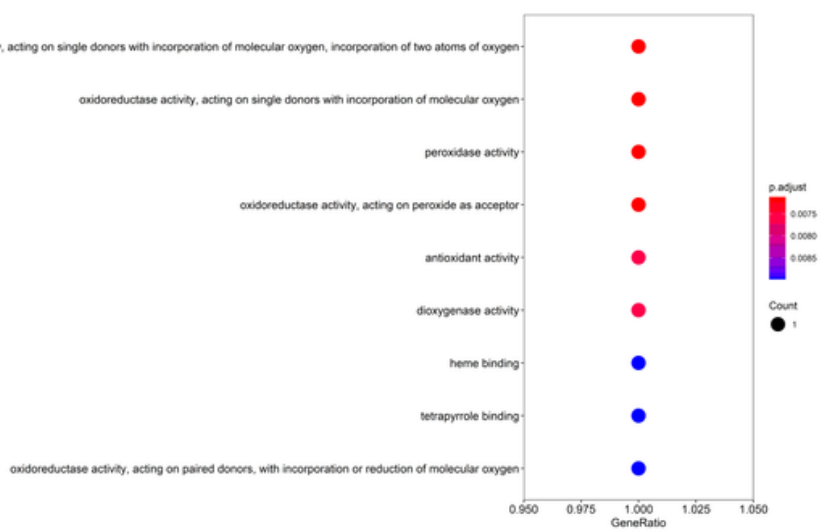

C

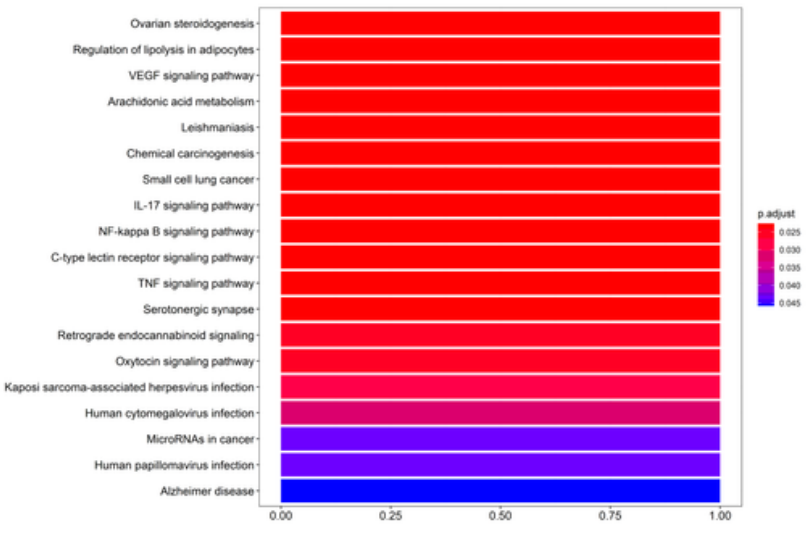

D

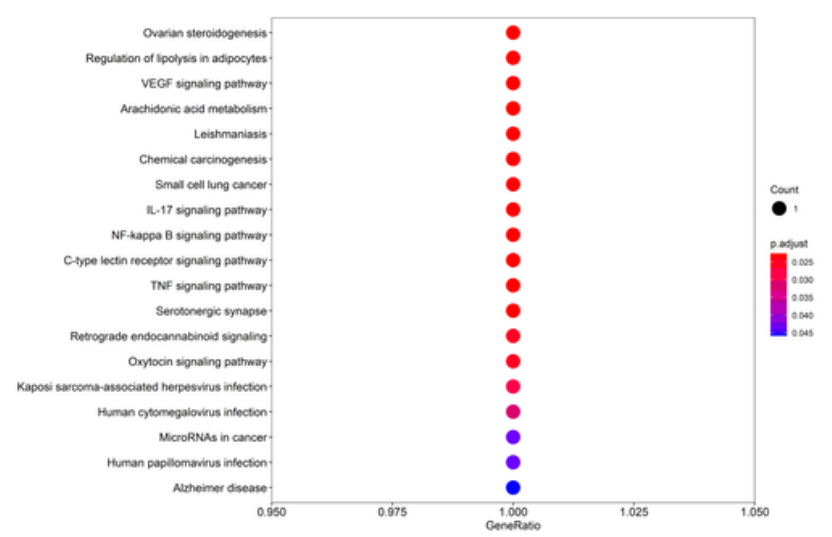

Figure 8

GO and KEGG enrichment analysis of targets at the intersection of etoricoxib and disease A. GO enrichment analysis histogram; B. Go enrichment analysis bubble diagram; C. KEGG enrichment analysis bar chart; D. KEGG enrichment analysis of bubbles 